\title{
Computational studies on the palladium-mediated oxidation of methanol to formaldehyde
}

Lucía Briones-Miguéns, ${ }^{a}$ Cristina Portela-García, ${ }^{a}$ Ana M. García-Deibe, ${ }^{a}$ Matilde Fondo, ${ }^{a}$ Jesús Sanmartín-Matalobos, ${ }^{a}$ Emilio Lence, ${ }^{b}$ Concepcion González-Bello. ${ }^{\text {b }}$

${ }^{a}$ Departamento de Química Inorgánica, Facultade de Química, Universidade de

Santiago de Compostela, E-15782 Santiago de Compostela, Spain.

E-mail: jesus.sanmartin@usc.es

${ }^{\mathrm{b}}$ Centro Singular de Investigacion en Química Biológica y Materiales Moleculares

(CIQUS), Universidad de Santiago de Compostela, Jenaro de la Fuente s/n, 15782

Santiago de Compostela, Spain.

\begin{abstract}
We have carried out computational studies on the metal-mediated oxidation of methanol to formaldehyde using $\mathrm{Pd}\left(\mathrm{L}_{\mathrm{SB}}\right) \cdot 3 \mathrm{H}_{2} \mathrm{O}$, where $\mathrm{L}_{\mathrm{SB}}$ is the dianionic form of the Schiff base ligand $N$-\{2-[(8-hydroxyquinolin-2-yl)methyleneamino]benzyl $\}-4-$ methylbenzenesulfonamide. With the aim of reducing the overall Gibbs energy of the global process, we have studied the replacement of hydroxymethoxymethyl by hydroxymethyl and aminomethyl groups at 2-position of the quinoline ring.
\end{abstract}

\section{Keywords}

DFT calculations / Methanol oxidation / Schiff base / Palladium

\section{Introduction}

Very recently, we have reported the unexpected metal-mediated aerobic oxidation of methanol to formaldehyde using $\mathrm{Pd}\left(\mathrm{L}_{\mathrm{SB}}\right) \cdot 3 \mathrm{H}_{2} \mathrm{O}$, where $\mathrm{L}_{\mathrm{SB}}$ is the dianionic form of the Schiff base ligand $N$-\{2-[(8-hydroxyquinolin-2-yl)methyleneamino]benzyl $\}-4-$ methylbenzenesulfonamide [1]. We have demonstrated that the reaction pathway would involve the acid-catalyzed hydrolysis of the Schiff base ligand in $\mathrm{Pd}\left(\mathrm{L}_{\mathrm{SB}}\right) \cdot 3 \mathrm{H}_{2} \mathrm{O}$ (Fig. 1).
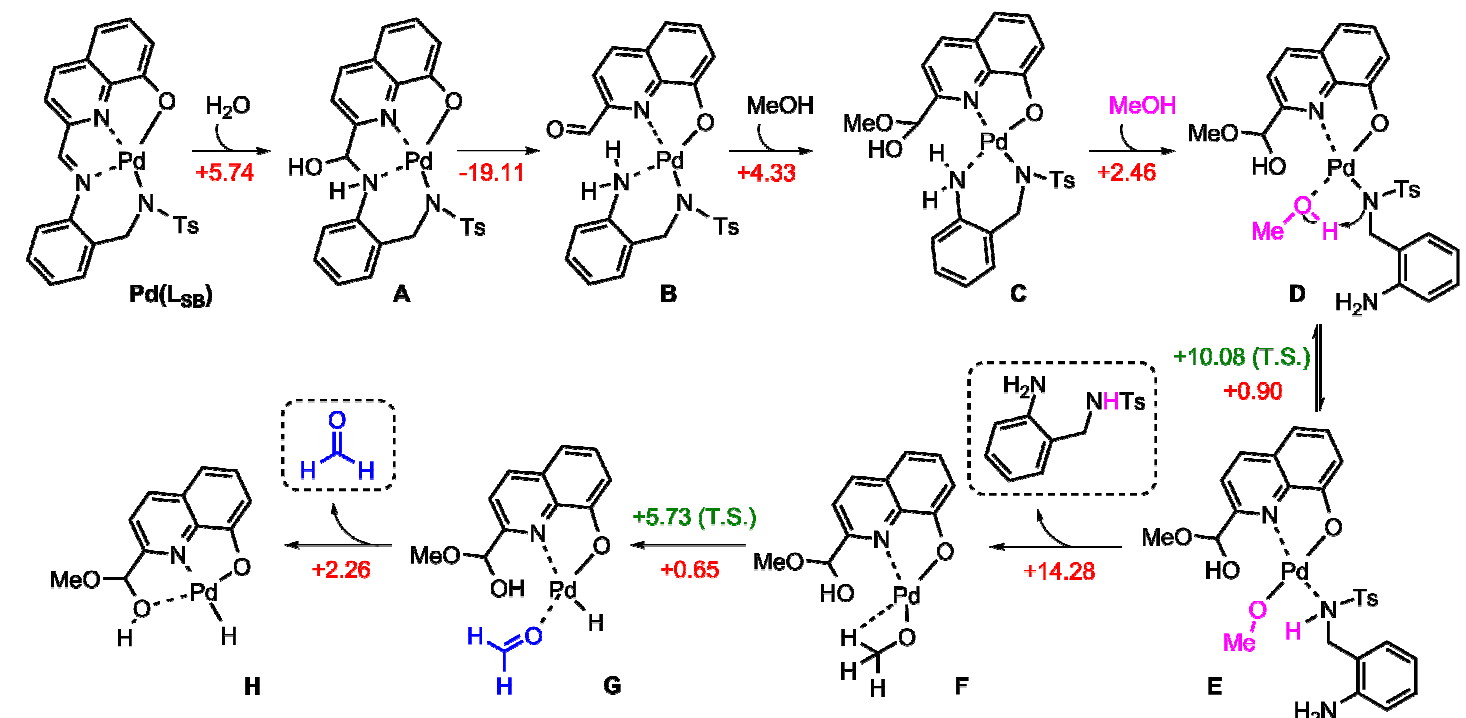

Fig. 1. Proposed pathway for the aerobic oxidation of methanol to formaldehyde using $\mathrm{Pd}\left(\mathrm{L}_{\mathrm{SB}}\right) \cdot 3 \mathrm{H}_{2} \mathrm{O}$ (solvated water was omitted for clarity). Calculated $\Delta \mathrm{G}$ values $\left(\mathrm{kcal} \mathrm{mol}^{-1}\right)$ for each step are indicated in red color. $E_{a}$ values $\left(\mathrm{kcal} \mathrm{mol}^{-1}\right)$ for $\mathbf{D} \rightarrow \mathbf{E}$ and $\mathbf{F} \rightarrow \mathbf{G}$ steps are indicated in green color. 
The hydrolysis of $\mathrm{Pd}\left(\mathrm{L}_{\mathrm{SB}}\right)$ would take place to afford the Pd-aldehyde complex $\mathbf{B}$ via the Pd-hydroxyamino complex A. Subsequent nucleophilic addition of methanol would afford the Pd-hemiacetal complex $\mathbf{C}$. The next step would involve the access to the palladium coordination sphere of a methanol molecule to give the Pd-methanol complex D. This occurs with breaking of the Pd- $\mathrm{N}_{\text {amine }}$ bond in C. Next, the intramolecular deprotonation of methanol would take place in $\mathbf{D}$ to give the $\mathrm{Pd}$ methoxide complex E. The next step would be the dissociation of the $\mathrm{Pd}-\mathrm{N}_{\text {sulfonamide }}$ bond that would liberate 2-tosylaminomethylaniline to the reaction medium and afford the Pd-alkoxide complex $\mathbf{F}$. The $\beta$-hydride elimination, which would take place in $\mathbf{F}$, gave the Pd-formaldehyde complex $\mathbf{G}$. The next step should be the dissociation of the $\mathrm{Pd}-\mathrm{O}_{\text {formaldehyde }}$ bond that would liberate formaldehyde to the reaction medium and afford the Pd-hydride complex $\mathbf{H}$.

From a thermodynamic point of view, a rate-limiting step is the deprotonation of methanol $\left(\mathbf{D} \rightarrow \mathbf{E}, E_{a}=10.08 \mathrm{kcal} . \mathrm{mol}^{-1}\right)$, but the most unfavorable step of the proposed pathway would correspond to the dissociation of the diamine ligand of the metal center $\left(\mathbf{E} \rightarrow \mathbf{F}, \Delta \mathrm{G}=14.28 \mathrm{kcal}^{\mathrm{mol}} \mathrm{m}^{-1}\right)$. Surprisingly, the $\beta$-elimination step $(\mathbf{F} \rightarrow \mathbf{G})$ has a low activation energy value $\left(E_{a}=5.73 \mathrm{kcal} . \mathrm{mol}^{-1}\right)$. With this data in hand, we considered that perhaps a reduction in the energy cost of these processes it could be achieved by increasing the binding ability of the quinoline ligand. Accordingly, we take a step further by studying the replacement of hydroxymethoxymethyl by hydroxymethyl and aminomethyl groups at 2-position of the quinoline ring to try to reduce the overall Gibbs energy of the global process.

\section{Experimental}

\section{Computational studies}

Geometry optimizations and energy calculations were performed using the Gaussian 09W program package [2] at the density functional theory (DFT) level by means of the hybrid B3LYP functional using the standard 6-31G(d) for $\mathrm{C}, \mathrm{H}, \mathrm{O}, \mathrm{S}$, and $\mathrm{N}$ and the LANZL2DZ basis sets relativistic pseudopotential for Pd.

The available crystal structure of $\mathrm{Pd}\left(\mathrm{L}_{\mathrm{SB}}\right) \cdot \mathrm{CH}_{2} \mathrm{Cl}_{2}$ [3], which was originated by recrystallization of $\mathrm{Pd}\left(\mathrm{L}_{\mathrm{SB}}\right) \cdot 3 \mathrm{H}_{2} \mathrm{O}$ from $\mathrm{CH}_{2} \mathrm{Cl}_{2}$, was used for these studies. Harmonic frequencies were calculated at the same level of theory to characterize the stationary points and to determine the zero-point energies (ZPEs). The calculations were performed in vacuum.

\section{Results and discussion}

We have analyzed the free Gibbs energy of the reactions involving palladium complexes from $\mathbf{C}$ to $\mathbf{H}$, after replacement of the hydroxymethoxymethyl group at 2position of the hydroxyquinoline moiety by hydroxymethyl and an aminomethyl groups (Table 1). Besides, $E_{a}$ values $\left(\mathrm{kcal} \mathrm{mol}^{-1}\right)$ for $\mathbf{D} \rightarrow \mathbf{E}$ and $\mathbf{F} \rightarrow \mathbf{G}$ steps were calculated.

Fig. 2 shows a comparison of the calculated free Gibbs energy resulted from the use of 2-hydroxymethyl-8-hydroxyquinoline ( $\left.\mathrm{HL}^{2}\right)$ and 2-aminomethyl-8hydroxyquinoline $\left(\mathrm{HL}^{3}\right)$ instead of 2-hydroxymethoxymethyl-8-hydroxyquinoline $\left(\mathrm{HL}^{1}\right)$. The theoretical studies using DFT calculations suggested that no substantial difference would produce the replacement of the hydroxymethoxymethyl group by a hydroxymethyl one over the formation of the Pd-hydride complex $\mathbf{H}$. However, replacement of the hydroxymethoxymethyl group by an aminomethyl one would have a significant effect over the dissociation of the 2-tosylaminomethylaniline ligand from 
the metal center (Table $1, \mathbf{E} \rightarrow \mathbf{F}$ step, calculated energy drop about $6 \mathrm{kcal}^{\mathrm{mol}} \mathrm{l}^{-1}$ ). Moreover, the $\beta$-elimination step would be now a thermodynamically favorable process with a $\Delta \mathrm{G}=-9.43 \mathrm{kcal}^{\mathrm{mol}}{ }^{-1}$ (Table $1, \mathbf{G} \rightarrow \mathbf{H}$ step). The deprotonation step is however about $4 \mathrm{kcal} \cdot \mathrm{mol}^{-1}$ less favorable (Table $1, \mathbf{D} \rightarrow \mathbf{E}$ step), but the activation energy of this process would be maintained the same value (10.96 kcal.mol ${ }^{-1}$ vs 10.08 kcal.mol ${ }^{-1}$ ).

Table 1. $\Delta \mathrm{G}$ for the reaction steps leading to $\mathbf{H}$ from $\mathbf{C}$ including two transition states (T.S.).

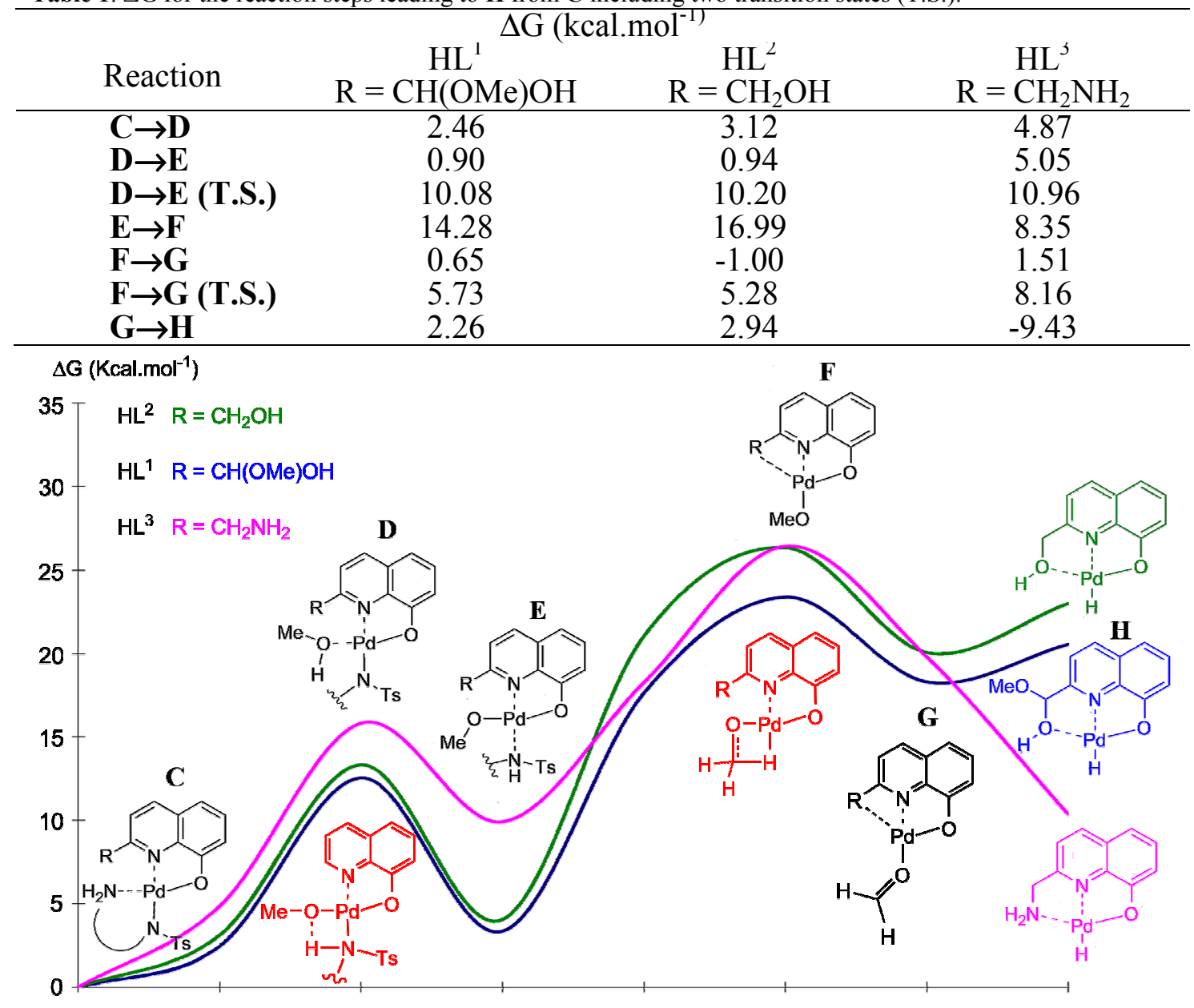

Fig. 2. Comparison of the Gibbs free energy of the steps involving the Pd-complexes from $\mathbf{C}$ to $\mathbf{H}$.

\section{Conclusions}

The use of 2-aminomethyl-8-hydroxyquinoline $\left(\mathrm{HL}^{3}\right)$ instead of 2hydroxymethoxymethyl-8-hydroxyquinoline $\left(\mathrm{HL}^{1}\right)$ leads to a reduction of the overall Gibbs energy of the palladium-mediated oxidation of methanol to formaldehyde, while no substantial difference would produce the use of 2-hydroxymethyl-8hydroxyquinoline $\left(\mathrm{HL}^{2}\right)$.

\section{References}

[1] J. Sanmartin-Matalobos, A. M. Garcia-Deibe, L. Briones-Miguéns, E. Lence, C. Gonzalez-Bello, C. Portela-Garcia and M. Fondo, New J. Chem., DOI: 10.1039/ c3nj00658a.

[2] Gaussian 09, Revision A.2, M. J. Frisch, G. W. Trucks, H. B. Schlegel, G. E. Scuseria, M. A. Robb, J. R. Cheeseman, G. Scalmani, V. Barone, B. Mennucci, G. 
A. Petersson, H. Nakatsuji, M. Caricato, X. Li, H. P. Hratchian, A. F. Izmaylov, J. Bloino, G. Zheng, J. L. Sonnenberg, M. Hada, M. Ehara, K. Toyota, R. Fukuda, J. Hasegawa, M. Ishida, T. Nakajima, Y. Honda, O. Kitao, H. Nakai, T. Vreven, J. A. Montgomery, Jr., J. E. Peralta, F. Ogliaro, M. Bearpark, J. J. Heyd, E. Brothers, K. N. Kudin, V. N. Staroverov, R. Kobayashi, J. Normand, K. Raghavachari, A. Rendell, J. C. Burant, S. S. Iyengar, J. Tomasi, M. Cossi, N. Rega, J. M. Millam, M. Klene, J. E. Knox, J. B. Cross, V. Bakken, C. Adamo, J. Jaramillo, R. Gomperts, R. E. Stratmann, O. Yazyev, A. J. Austin, R. Cammi, C. Pomelli, J. W. Ochterski, R. L. Martin, K. Morokuma, V. G. Zakrzewski, G. A. Voth, P. Salvador, J. J. Dannenberg, S. Dapprich, A. D. Daniels, Ö. Farkas, J. B. Foresman, J. V. Ortiz, J. Cioslowski, and D. J. Fox, Gaussian, Inc., Wallingford CT, 2009.

[3] A. M. Garcia-Deibe, J. Sanmartin-Matalobos, C. Gonzalez-Bello, E. Lence, C. Portela-Garcia, L. Martinez-Rodriguez and M. Fondo, Inorg. Chem., 2012, 51, 1278. 